\title{
Corrigendum: A single amino acid residue can determine the sensitivity of SERCAs to artemisinins
}

\author{
Anne-Catrin Uhlemann, Angus Cameron, Ursula Eckstein-Ludwig, Jorge Fischbarg, Pavel Iserovich, Felipe A Zuniga, Malcolm East, \\ Anthony Lee, Leo Brady, Richard K Haynes \& Sanjeev Krishna \\ Nat. Struct. Mol. Biol. 12, 628-629 (2005); published online 5 June 2005; corrected after print 3 February 2012
}

Note: U.E.-L. was unavailable to comment on this corrigendum.

In our original study, we concluded that mutations in Plasmodium falciparum ATP6 (PfATP6) can alter sensitivity to artemisinins when the protein is expressed in Xenopus laevis oocytes. We became aware that some experimental data points had not been included in the original analysis, and the method used to derive the $K_{\mathrm{i}}$ values in Table 1 was not standardized. To address these issues, we reassessed the original data, after standardizing the analytical procedures and parameters used in nonlinear analysis (Supplementary Methods). Although missing data points were included for some experiments, the data for a few experiments could not be retrieved. This reanalysis confirms our previous conclusion that single amino acid mutations in PfATP6 can abolish sensitivity to artemisinins, as shown by the results obtained with PfATP6 mutants L263D, L263E and L263K (Supplementary Fig. 1). On the other hand and in contrast to what we originally reported, the variability and magnitude of signal to noise in the $\mathrm{Ca}^{2+}$-ATPase activities of other mutants do not always allow us to reliably derive $K_{\mathrm{i}}$ values (Supplementary Fig. 2 and Supplementary Table). This affects the results reported for PfATP6 mutants L263A, L263S, L263Q, F264S and I89T, mammalian SERCA1 E225L, PvSERCA and PbSERCA.

Signal to noise can vary considerably in this system in individual experiments as a function of background activity, batch of oocytes, amount of expressed transporter and other variables. These may all contribute to errors that can be relatively large in individual experiments. Graphs for individual estimations of apparent $K_{\mathrm{i}}$ values are shown in Supplementary Figure 2. Only one experiment could be reanalyzed for the mammalian SERCA E255L mutation. Despite these limitations, larger (or aggregated) datasets indicate that differences could be observed between wild-type PfATP6 and some mutants, suggesting the intermediate sensitivity of the latter (for example, L263A and L263S), and between PfATP6 and PvSERVA or PbSERCA. Further studies (unpublished data) suggest that increasing the quantity of protein expressed in oocyte membranes, which would reduce assay time, and confirming the adequate expression of PfATP6 by preliminary studies with inhibitor can improve signal to noise and hence the reliability of these assays. However, the experimental data from the original study alone do not allow us to support reliable $K_{\mathrm{i}}$ values as originally claimed.

The Supplementary Information associated with this corrigendum can be found at http://www.nature.com/nsmb/journal/v19/n2/extref/ nsmb0212-264-S1.pdf. 